\title{
Image Steganography Method to Improve the Stego Image Quality and Security using Reversible Texture Synthesis
}

\author{
V. Lokeswara Reddy, PhD \\ M.Tech. \\ Associate Professor \\ Department of CSE, \\ KSRM College of Engineering, \\ Kadapa, \\ YSR District, A.P (INDIA)
}

\author{
K. Tejaswi \\ Post Graduate Student \\ Department of CSE \\ KSRM College of Engineering, \\ Kadapa, \\ YSR District, A.P (INDIA)
}

\begin{abstract}
Steganography is the profession of concealing a privacy message within a stated medium like image, audio or video. The main issue regarding every steganography technique is the changes that occur in the cover image after the data embedding procedure. We are using the source texture image for embedding privacy message in diversion with the cover image. This technique grants us to de-embedding the privacy message and source texture separating the stego synthetic texture. The advantages offering from this technique are embedding capacity that is symmetrical to the size of the stego texture image and the reversible potency to recover the source texture. The texture synthesis method is nothing but the generating a new large texture image with same local appearance and variable size from the small size image.
\end{abstract}

\section{Keywords}

Steganography, Texture Synthesis, Cover Image, Stego Image.

\section{INTRODUCTION}

Steganography is the profession of concealing a privacy message within a stated medium like image, audio or video. The term steganography is gathered from the two greek terms "steganos" and "grapheia" means "protected" and "writing" consequently. The purpose of steganography is to establish communication between two individuals whose privacy messages is unknown to third party individual.

Today's image steganographic methods use an host image as a cover image. The result of using these host images as cover image leads to image distortion in stego image. The image distortion in the stego image causes the deficiency of size of the cover image is stable. This leads to more image distortion if we embed more privacy messages; security is also major issue in this method of using host image as cover image.

In this paper we present a novel approach image steganography method to improve the stego image quality and security using reversible texture synthesis. The texture synthesis is nothing but re-sizes a smaller texture image which is taken as the cover image in order to generate a large texture image with identical local appearance and variable size. Instead of using host image as cover image we are using source texture image to embed privacy message by the method of texture synthesis. This method provides us to deembed the privacy message and the source texture from the stego synthetic texture. This paper offers the benefits of the size of the cove image is enlarged i.e. up to the size of the stego texture image and the reversible technique in this method offers the functionality to recover the source texture.

\section{LITERATURE SURVEY}

Texture synthesis has acquired a large immersion in computer vision and computer graphics [1]. The modern work has concentrated on texture synthesis. By example in which a source texture image is re-sampled using either pixel based or patch based algorithms to generate a new synthesized texture image with same local appearance and variable size.

Pixel based algorithms [2, 4] produce the synthesized image pixel by pixel and use spatial neighborhood comparisons to pick the most parallel pixel in a sample texture as the output pixel. Since each output pixel is resolved by the earlier synthesized pixels, any wrongly synthesized pixels meanwhile in the procedure significance the remaining result creating propagation of errors.

Otori and kuriyama $[5,6]$ begin the process of united data coding with pixel based texture synthesis. Privacy message to be covered are embedded into colored dotted pattern and they are absolutely painted on a blank image. A pixel based algorithm hide the remaining pixels by the pixel based texture synthesis procedure, thus hiding the presence of dotted patterns. To de-embed the messages the printout of the stego synthesized texture image is photographed previously applying the data detecting methods. The capacity accommodated by the authors in this method is based upon the number of dotted patterns. However, their method had a minute error rate during the message de-embedding.

Patch based algorithms [7, 8] attach patches from a source texture on behalf of pixel to synthesize textures. The approach of Cohen et al and $\mathrm{Xu}$ et al. increases the image quality of pixel based synthetic because texture structures inside the patches are prolonged. However since patches are prefixed with a small overlapped area during the synthetic process, one charges to make a push to ensure that the patches admit with their neighbors.

Liang et al. [9] implemented the patch-based sampling procedure and used the heeling approach for the fold over areas of neighboring patches. Efros and freeman [10] introduced a patch stitching method called "image quilting". For each advanced patch to be incorporate and baste, the algorithm first watches the source texture and picks one candidate patch that brighten up the pre-defined error compassion with respect to neighbors along the overlapped area. After words, a dynamic programming method is utilized 
to exhibit the minimum error patch through the overlapped area. This tells an optimal boundary between the picked candidate patch and the synthesized patch, generating visually credible patch stitching.

$\mathrm{Ni}$ et al. [11] presented a data hiding algorithms which can deembed the cover image without any changes from the stego image after the privacy message have been de-embedded. Histogram shifting is a suggestible technique among the existed ones of reversible image data hiding because it can rule the changes to pixels, thus decreasing the embedding distortion, and it only takes a small size location map, thereby decreasing the overhead encountered.

In this paper, we are taking the benefits of patch-based methods to embed a privacy message during the synthesizing process. This provides the source texture to be returned in a message extracting process, allowing the functionality of reversibility.

\section{METHODOLOGY}

The proposed method is illustrated in this section. The basic unit in the stegonagraphic texture synthesis is involved to as a "patch". The patch is stand for an image block of a source texture where its size is specified by the user. The combination of patches gives the composition image in which the privacy message is embedded. This paper contains mainly two steps:

\section{Procedure for Message Embedding}

2. Source texture Recovery, Message Extraction \& Message Authentication procedure

1. Procedure for Message Embedding:

The steps involved in this procedure are
a. Index Table Generation
b. Patch composition process
c. Message oriented texture synthesis process

\section{Index table generation}

The first step involved in this paper is index table generation where we will generate an index table to store the location of the source patch in the synthetic texture. The index table gives us to access the synthetic texture \& retrieve the source texture totally. The user specified texture size is generated by using this index table.

\section{Patch composition process}

The second step in the project is to stick the source patches into a workbench to generate the composition image. First we take the empty image as our workbench where the size of the workbench is similar to that of synthetic texture. According to the source patch ID's stored in the index table; we then stick the source patches into the workbench. During the sticking process, if no overlapping is occurred between the source patches then we can directly planted the source patch into the workbench

\section{Message oriented texture synthesis process:}

After generating the composition image we have to embed the privacy message by the process of message oriented texture synthesis to produce the final stego synthetic texture.

\section{Source Texture Recovery, Message Extraction and Message Authentication procedure:}

The de-embedding done at the receiver side involves creating the index table, regain the source texture, performing the texture synthesis, extracting \& authenticating the privacy message present inside the stego synthetic texture.

\section{EXPERIMENTAL WORK}

The following Figure 1 shows the homepage. The login and registration procedure is done in this home page.

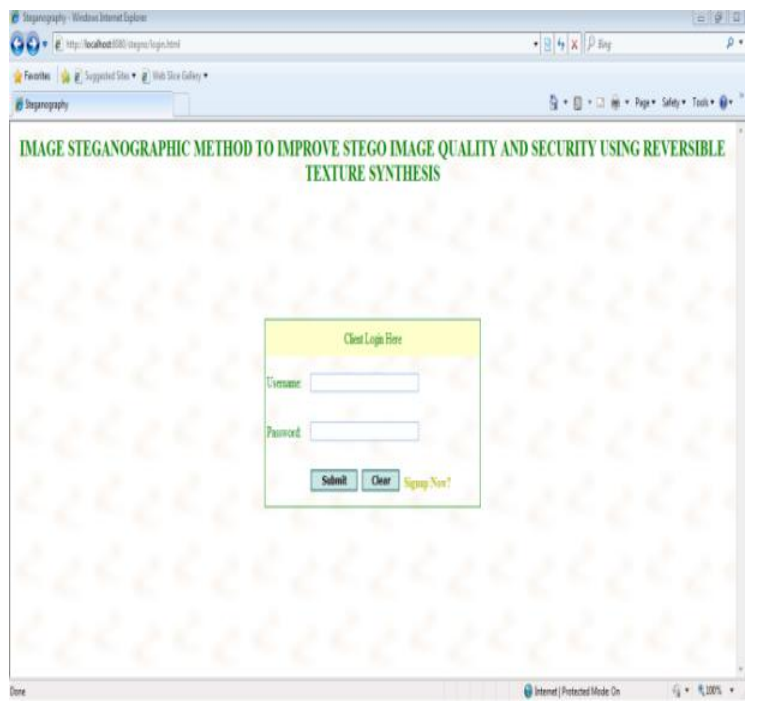

Fig 1: Home page

The figure of encoding procedure is shown in the following Figure 2. First we have to select the image which we want to embed the data.

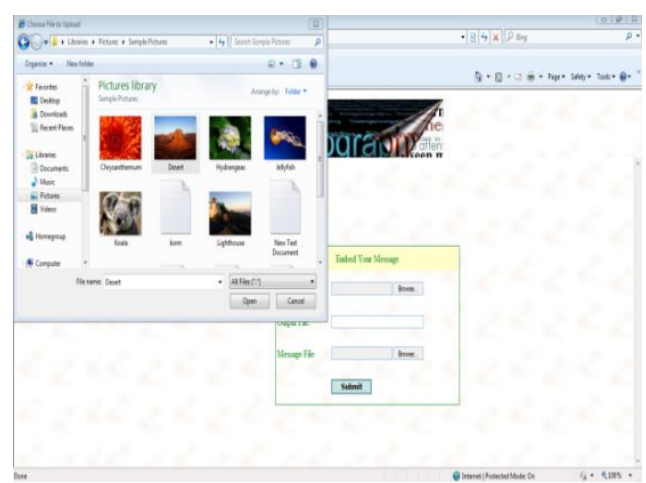

Fig 2:Encoding process at sender side

The Figure 3 shows the entering of secret data for embedding into cover image.

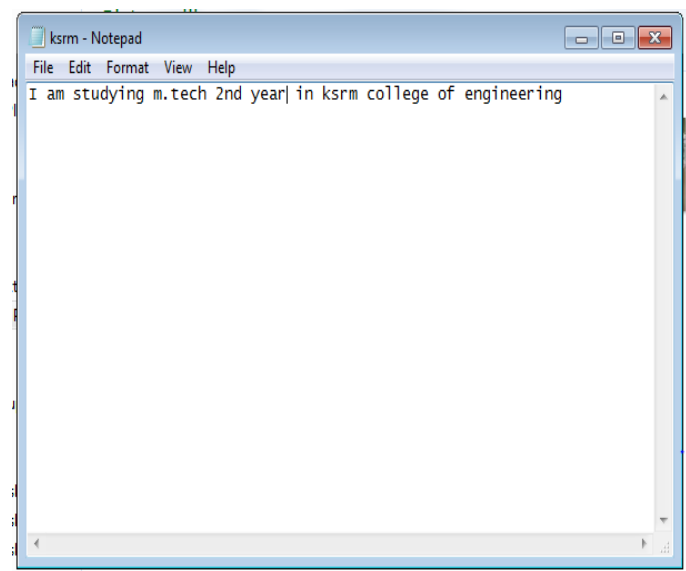

Fig 3: Secret Information Entered at sender side 
Then we have to generate an index table for the above text document. If the data is present then it displays " 1 " in the index table if the data is not present then it displays " -1 ".

The index table is shown in the following Figure 4

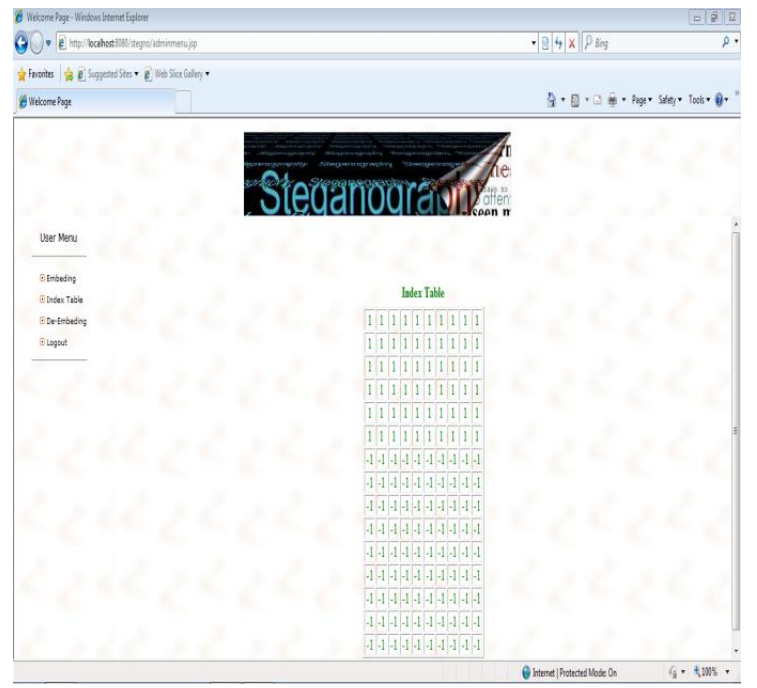

Fig 4: Index Table

The de-embedding process at receiver side is shown in the figure 5

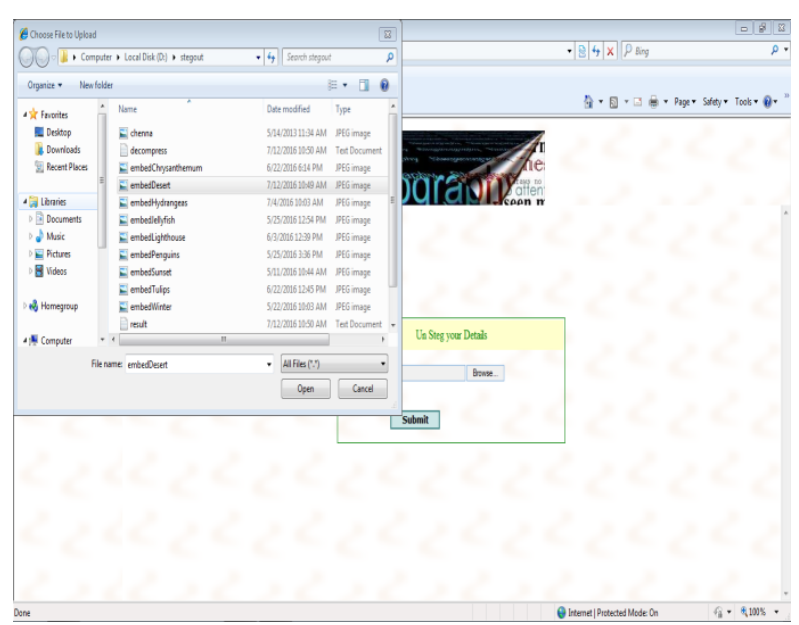

Fig 5: De-embedding process at Receiver side

\section{CONCLUSION}

This paper proposes a reversible steganographic method using texture synthesis. By giving the original source texture, our method generates a large stego synthetic texture for embedding the privacy message. we used the conventional patch based texture synthesis. The proposed method is novel and gives reversibility to retake the original source texture from the stego synthetic textures, making possible to provide another round of texture synthesis if needed. The proposed method gives us the high quality stego image as well as high security.

\section{REFERENCES}

[1] Y. Guo, G. Zhao, Z. Zhou, and M. Pietikäinen, "Video texture synthesis with multi-frame LBP-TOP and diffeomorphic growth model," IEEE Trans. Image Process., vol. 22, no. 10, pp. 3879- 3891, Oct. 2013.

[2] L.-Y. Wei and M. Levoy, "Fast texture synthesis using treestructured vector quantization," in Proc. 27th Annu. Conf. Comput. Graph. Interact. Techn, 2000, pp. 479 488 .

[3] A. A. Efros and T. K. Leung, "Texture synthesis by nonparametric sampling," in Proc. 7th IEEE Int. Conf. Comput. Vis., Sep. 1999, pp. 1033-1038.

[4] C. Han, E. Risser, R. Ramamoorthi, and E. Grinspun, "Multiscale texture synthesis," ACM Trans. Graph., vol. 27, no. 3, 2008, Art. ID 51.

[5] H. Otori and S. Kuriyama, "Data-embeddable texture synthesis," in Proc.8th Int. Symp. Smart Graph, Kyoto, Japan, 2007, pp. 146-157.

[6] H. Otori and S. Kuriyama, "Texture synthesis for mobile data communications,"IEEE Comput. Graph. Appl., vol. 29, no. 6, pp. 74-81, Nov. /Dec. 2009.

[7] M. F. Cohen, J. Shade, S. Hiller, and O. Deussen, "Wang tiles for image and texture generation," ACM Trans. Graph., vol. 22, no. 3, pp. 287-294, 2003.

[8] K. Xu et al., "Feature-aligned shape texturing," ACM Trans. Graph., vol. 28, no. 5, 2009, Art. ID 108. [9] L. Liang, C. Liu, Y.-Q. Xu, B. Guo, and H.-Y. Shum, "Real-time texture synthesis by patch-based sampling," ACM Trans. Graph., vol. 20, no. 3, pp. 127-150, 2001.

[9] A. A. Efros and W. T. Freeman, "Image quilting for texture synthesis and transfer," in Proc. 28th Annu. Conf. Comput. Graph. Interact. Techn, 2001, pp. 341-346.

[10] Z. Ni, Y.-Q. Shi, N. Ansari, and W. Su, "Reversible data hiding," IEEE Trans. Circuits Syst. Video Technol., vol. 16, no. 3, pp. 354-362,Mar.2006.

\section{AUTHOR PROFILE}

Dr. V. Lokeswara Reddy received his $\mathrm{Ph}$. D in Computer Science and Engineering from JNTUA, Ananthapuramu in the year 2015. Received his M. Tech (CSE) degree from SRM University, Chennai in the year 2005. Received his M.C.A degree from S.V. University, Tirupati in the year 2000. He has a total of 15 years of experience in teaching. Currently he is working as Associate Professor in CSE Department at K.S.R.M College of Engineering, Kadapa. He has presented 10 papers in International, National Conferences and published 17 papers in International journals.

K..Tejaswi received her B.Tech degree in Computer Science and Engineering from JNTUA, Ananthapuramu in the year 2014. She is pursuing her M.Tech at K.S.R.M College of Engineering, Kadapa (Autonomous) Andhra Pradesh. Her current research interests include hiding techniques. 